\title{
PRE-OPERATIVE ERCP BILE DUCT STENTING AND LAPAROSCOPIC CHOLEDOCHOPLASTY IS SAFE AND EFFECTIVE IN THE MANAGEMENT OF MIRIZZI'S SYNDROME
}

\author{
Bodaiah Talamala ${ }^{1}$ K. Jagan Mohan Rao², G. Nagabhusanam³, U. Bhaskar Rao ${ }^{4}$, Varun Dasari ${ }^{5}$
}

${ }^{1}$ Consultant Surgical Gastroenterologist, Department of Medical and Surgical Gastroenterology, Nagarjuna Hospital, Kanuru, Vijayawada, Andhra Pradesh India.

${ }^{2}$ Consultant Medical Gastroenterologist, Department of Medical and Surgical Gastroenterology, Nagarjuna Hospital, Kanuru, Vijayawada, Andhra Pradesh India.

${ }^{3}$ Consultant Medical Gastroenterologist, Department of Medical and Surgical Gastroenterology, Nagarjuna Hospital, Kanuru, Vijayawada, Andhra Pradesh India.

${ }^{4}$ Consultant Medical Gastroenterologist, Department of Medical and Surgical Gastroenterology, Nagarjuna Hospital, Kanuru, Vijayawada, Andhra Pradesh India.

${ }^{5}$ Consultant Surgical Gastroenterologist, Department of Medical and Surgical Gastroenterology, Nagarjuna Hospital, Kanuru, Vijayawada, Andhra Pradesh India.

\begin{abstract}
\section{BACKGROUND}

Mirizzi's syndrome is a rare condition caused by the obstruction of the common bile duct or common hepatic duct by external compression from multiple impacted gallstones or a single large impacted gallstone in Hartman's pouch. Presenting symptoms are similar to cholecystitis but may be confused with other obstructing conditions such as common bile duct stones and ascending cholangitis due to presence of jaundice. Preoperative diagnosis is often difficult and usually missed. We wanted to analyse the clinical presentation, pre-operative diagnostic strategies, pre-operative bile duct stenting with ERCP, operative management and outcome of patients operated for Mirizzi's syndrome in a tertiary care center.
\end{abstract}

\section{METHODS}

This is retrospective study. Patients operated for Mirizzi's syndrome between May 2015 and August 2018 were included in the study. Their pre-operative demographics, pre-operative diagnostic strategies, operative management and outcome were recorded and analysed.

\section{RESULTS}

A total of 6 patients was identified out of 350 laparoscopic cholecystectomies performed during study period giving an incidence of $1.7 \%$. There were 5 males and 1 female with a mean age of 50 years. abdominal pain and jaundice were predominant symptoms and altered liver function tests were seen in all patients. Magnetic resonance cholangiopancreatography (MRCP) was the main stay of diagnosis and diagnostic of Mirizzi's syndrome in all patients. All patients in this study were having type III Mirizzi's syndrome. Pre-operative endoscopic retrograde cholangiopancreatography (ERCP), bile duct stenting, and laparoscopic choledochoplasty sufficed in all patients and none required Hepaticojejunostomy.

\section{CONCLUSIONS}

Mirizzi's syndrome a rare complication of cholelithiasis is a formidable diagnostic and therapeutic challenge. Pre-operative Magnetic Resonance Cholangiopancreatography (MRCP) is the main diagnostic strategy. Preoperative Endoscopic Retrograde Cholangiopancreatography (ERCP), bile duct stenting enable the surgeon to identify and minimize duct injury, and laparoscopic choledochoplasty is feasible and safe in most cases as well.

HOW TO CITE THIS ARTICLE: Talamala BK. Rao KJM, Nagabhusanam G, R, et al. Pre-operative ERCP bile duct stenting and laparoscopic choledochoplasty is safe and effective in the management of Mirizzi's syndrome. J. Evolution Med. Dent. Sci. 2019;8(15):1240-1242, DOI: $10.14260 /$ jemds/2019/275

\section{BACKGROUND}

Mirizzi's syndrome (MS) is an unusual complication of gallstone disease and occurs in approximately $1 \%$ of all patients with cholelithiasis.[1] The syndrome was first described in 1948 and is characterized by impaction of stones in the cystic duct or neck of the gallbladder (GB), resulting in mechanical obstruction of the common hepatic duct and frequent clinical presentation of intermittent or constant jaundice.

'Financial or Other Competing Interest': None.

Submission 18-03-2019, Peer Review 05-04-2019,

Acceptance 09-04-2019, Published 15-04-2019.

Corresponding Author:

Dr. Bodaiah Talamala,

Consultant Surgical Gastroenterologist,

Nagarjuna Hospital, Kanuru, Vijayawada-520001,

Andhra Pradesh, India.

E-mail:4750200@gmail.com

DOI: $10.14260 /$ jemds $/ 2019 / 275$

\section{(c) $($ ) $\$$}

The majority of cases are not identified preoperatively, despite the availability of modern imaging techniques. The purpose of this study is to analyse the pre-operative diagnostic methods, operative strategies and outcomes of surgical treatment in patients with Mirizzi's syndrome.

\section{METHODS}

This is a retrospective analysis that evaluated patients diagnosed with Mirizzi's syndrome during the period from May 2015 to August 2018. The patient's demographic variables, clinical presentation, laboratory findings, diagnostic modalities, presence of choledocholithiasis, therapeutic procedures, postoperative complications, and follow-up period were evaluated. The Csendes classification was followed to categorize these patients based on pre-operative and intra-operative findings. All patients were seen in the Medical/ surgical gastroenterology department within 3 months from their discharge from the hospital and every 6 months thereafter. 


\section{RESULTS}

During this period, 350 cholecystectomies were performed in our department, among which 6 patients were diagnosed with MS yielding an incidence about $1.7 \%$. There were 1 female and 5 males with a mean age of 50 years. All patients presented with abdominal pain, and jaundice, the mean duration of symptoms being 15 days. Liver function tests were altered in all patients, the elevated bilirubin and serum glutamic oxaloacetic transaminase, serum glutamic-pyruvic transaminase, and alkaline phosphatase (ALP) of all patients. Ultrasound abdomen the initial investigation of choice was diagnostic of Mirizzi's syndrome in 2 patients. Dilatation of intrahepatic biliary radicals, and proximal common bile duct (CBD), and non-visualized distal CBD, are features suggestive of Mirizzi's syndrome. Magnetic resonance cholangiopancreatography (MRCP) done in all patients was diagnostic in $100 \%$. endoscopic retrograde cholangiopancreatography (ERCP) bile duct stenting done in all patients ,laparoscopic choledochoplasty was done in all patients, laparoscopic ports are kept respectively as normal laparoscopic cholecystectomy, all cases having dense pericholecystic adhesions and frozen Calot's triangle, after releasing of adhesions gallbladder opened at Hartman's pouch, impacted stone was removed, after removal of stone bile duct was identified, stent noticed in bile duct ,Hartman's pouch was sutured over bile duct defect with 2.0 Vicryl interrupted sutures, remaining gallbladder removed,drain kept in all cases

The immediate post-operative course was uneventful in all patients. One patient had minor bile leak as evidenced by drain output, this was managed conservatively, and the leak subsided over a period of 4 days. All patents discharged at $5^{\text {th }}$ post-operative day. During follow-up, one patient was diagnosed with choledocholithiasis, underwent ERCP, and CBD clearance.

\section{DISCUSSION}

Mirizzi's syndrome is a rare cause of obstructive jaundice produced by the impaction of a gallstone either in the cystic duct or in the GB, resulting in stenosis of the extra hepatic bile duct, and in severe cases, direct cholecysto-choledochal fistula formation.[1] The reported incidence has range between $0.05 \%$ to $4 \%$ of all patients undergoing surgery for cholelithiasis.[2]

Its classification has evolved from Mc Sherry's original classification of two types to the four types as classified by Csendes.[2,3] Clinical or pre-operative diagnosis is quite difficult. Pain is the most common symptom reported in 54$100 \%$ of patients, followed by jaundice in 24 to $100 \%$ of patients, which was also the case in our series.[3]

Biochemical parameters the liver function tests show a cholestatic pattern, simulating those of choledocholithiasis. Serum bilirubin ranges from normal to as high as $12 \mathrm{mg} / \mathrm{dl}$ with a mean of 7-10 mg/dl and serum ALP levels range from normal to about three- to ten-fold rise. Imaging studies are however the mainstay of pre-operative diagnosis. ${ }^{[4,5]}$

Ultrasound scan abdomen has a sensitivity of $23-46 \%$ in detecting MS, suggestive findings include a small contracted Gall bladder, containing a stone in the cystic duct, intrahepatic, and proximal extra hepatic ductal dilatation, calculus in the common duct, and normal-calibre or unprofiled distal common duct.[5] CT scan has a sensitivity similar to US, it may show a characteristic irregular cavity adjacent to the neck of GB containing the protruding stone, but can be helpful in diagnosing other causes of obstructive jaundice such as Gall Bladder cancer, cholangiocarcinoma or metastatic tumour.

Pre-operative diagnosis is most important to avoid complications. Cholangiography remains the most reliable method of diagnosis of Mirizzi's syndrome, ERCP being the gold standard; the typical findings, include an excavating defect on the lateral wall of the CBD at the level of the cystic duct or Gall Bladder neck, dilated common hepatic and intrahepatic ducts and normal calibre CBD. The advantage with ERCP is its additional therapeutic role in clearing concomitant bile duct stones and insertion of a biliary stent or nasobiliary catheter both of which may serve a temporising role and may help in intra-operative identification of the CBD. $[2,6]$ Magnetic resonance particularly T2-weighted images can detect all the diagnostic components of Mirizzi's syndrome and can exclude malignancy on cross sectional imaging, while MRCP is equivalent to ERC in delineation of the ductal anatomy including the presence of fistula.[2,7]

The role of laparoscopic surgery in MS remains controversial and is considered technically challenging placing the patient at probably unnecessary increased risk of bile duct injuries. Several authors have reported high conversion rates and procedure related complication and as a consequence recommended that laparoscopic cholecystectomy should be undertaken in select cases of Mirizzi's Type I only, this based on the fact that a frozen Calot's triangle makes dissection hazardous at laparoscopy and unlike routine cases lateral traction on the infundibulum of Gall Bladder does not open up the Calot's triangle. $[4,6,8,9,10,11]$ A step by step approach to the laparoscopic management has been proposed by Rohatgi and Singh, highlighting the initial section of the GB fundus and retrieval of the impacted calculus to identify the infundibulum and cystic duct from thus facilitating a subtotal cholecystectomy.[12] However, the significance of a pre-operative diagnosis in lowering the risk of conversion and procedure related complications has also been highlighted by a few of the same authors, In fact, in our series a pre-operative suspicion of Mirizzi's syndrome at ERCP, along with the fact that these patients had a stent placed in the CBD before surgery enabled us to identify the CBD at surgery, and facilitated our dissection, thus lowering the risk of bile duct injuries. As suggested by Rohatgi and Singh our dissection concentrated on identifying the GB cystic duct junction and cystic duct CBD junction so that we were able to clear the impacted calculus from within and complete the cholecystectomy, while we resorted to a choledochotomy to retrieve the calculus from the cystic duct- CBD junction and the stent enabled us to achieve primary closure of the CBD in every case.[12,13] Chowbey et al. as early as 2000 had reported on a similar strategy and shown a good success rate with six conversions out of a total 22 cases in their series. ${ }^{[14]} \mathrm{A}$ similar successful combined endoscopic and laparoscopic approach has been published by Zheng et al., they stressed that these procedures should be performed by experienced hands. ${ }^{[15]} \mathrm{A}$ recent series by Li B et al. showed that a combined "tripartite" approach of pre-operative ERCP, laparoscopy, and intraoperative choledochoscope is a safe and effective means of treating Mirizzi's syndrome and is associated with faster post- 
operative recovery in terms of early resumption of oral feeds and shorter hospitalisation time.

\section{CONCLUSIONS}

Mirizzi's syndrome is a rare complication of long standing cholelithiasis that presents with features of choledocholithiasis. A pre-operative identification of the presence of Mirizzi's syndrome helps in operative planning, and this is facilitated by ERCP and MRCP, Pre-operative ERCP and bile duct stenting enable the surgeon to identify the bile duct and achieve a primary closure of the bile duct. As shown in our series, closure of CBD with a cuff of GB can be safely done even in Types III and IV Mirizzi's syndrome, with acceptable long tern outcomes. We also reiterate that a laparoscopic choledochoplasty is feasible and safe in an otherwise difficult clinical problem and is associated with good short-term recovery.

\section{REFERENCES}

[1] Mithani R, Schwesinger WH, Bingener J, et al. The Mirizzi's syndrome: Multidisciplinary management promotes optimal outcomes. J Gastrointest Surg 2008;12(6):1022-8.

[2] Ibrarullah M, Mishra T, Das AP. Mirizzi's syndrome. Indian J Surg 2008;70(6):281-7.

[3] Csendes A, Diaz JC, Burdiles P, et al. Mirizzi's syndrome and cholecystobiliary fistula: a unifying classification. Br J Surg 1989;76(11):1139-43.

[4] Ibrarullah MD, Saxena R, Sikora SS, et al. Mirizzi's syndrome identification and management strategy. Aust N Z J Surg 1993;63(10):802-6.

[5] Mishra MC, Vashishtha S, Tandon R. Biliobiliary fistula: pre-operative diagnosis and management implications. Surgery 1990;108(5):835-9.
[6] Choi BW, Kim MJ, Chung JJ, et al. Radiologic findings of Mirizzi's syndrome with emphasis on MRI. Yonsei Med J 2000;41(1):144-6.

[7] Beltran MA. Mirizzi's syndrome: History, current knowledge and proposal of a simplified classification. World J Gastroenterol 2012;18(34):4639-50.

[8] Aydin U, Yazici P, Ozsan I, et al. Surgical management of Mirizzi's syndrome. Turk J Gastroenterol 2008;19(4):258-63.

[9] Antoniou SA, Antoniou GA, Makridis C. Laparoscopic treatment of Mirizzi's syndrome: a systematic review. Surg Endosc 2010;24(1):33-9.

[10] Cui Y, Liu Y, Li Z, et al. Appraisal of diagnosis and surgical approach for Mirizzi's syndrome. ANZ J Surg 2012;82(10):708-13.

[11] Kwon AH, Inui H. Pre-operative diagnosis and efficacy of laparoscopic procedures in the treatment of Mirizzi's syndrome. J Am Coll Surg 2007;204(3):40915.

[12] Rohatgi A, Singh KK. Mirizzi's syndrome: Laparoscopic management by subtotal cholecystectomy. Surg Endosc 2006;20(9):1477-81.

[13] Singh K, Ohri A. Anatomic landmarks: their usefulness in safe laparoscopic cholecystectomy. Surg Endosc 2006;20(11):1754-8.

[14] Chowbey PK, Sharma A, Mann V, et al. The management of Mirizzi's syndrome in the laparoscopic era. Surg Laparosc Endosc Percutan Tech 2000;10(1):11-4.

[15] Zheng M, Cai W, Qin M. Combined laparoscopic and endoscopic treatment for Mirizzi's syndrome. Hepatogastroenterology 2011;58(109):1099-105. 